\title{
Article \\ Active Modulating the Intensity of Bifocal Metalens with Electrically Tunable Barium Titanate (BTO) Nanofins
}

\author{
Shuai Qin ${ }^{1}$, Hui Huang ${ }^{1}$, Kaiqian Jie ${ }^{1}$, Sirui Zeng ${ }^{2}$, Li Chen ${ }^{1}$, Hongzhan Liu ${ }^{1}$, Jianping Guo ${ }^{1}{ }^{1}$, \\ Hongyun Meng ${ }^{1}\left(\mathbb{0}\right.$, Faqiang Wang ${ }^{1}\left(\mathbb{D}\right.$, Xiangbo Yang ${ }^{1}$ and Zhongchao Wei ${ }^{1, *} \mathbb{1}$ \\ 1 Guangdong Provincial Key Laboratory of Nanophotonic Functional Materials and Devices, \\ School of Information and Optoelectronic Science and Engineering, South China Normal University, \\ Guangzhou 510006, China; shuaiqin@m.scnu.edu.cn (S.Q.); huihuang@m.scnu.edu.cn (H.H.); \\ kqjie@m.scnu.edu.cn (K.J.); lichen@m.scnu.edu.cn (L.C.); lhzscnu@163.com (H.L.); guojpgz@163.com (J.G.); \\ hymeng@scnu.edu.cn (H.M.); fqwang@scnu.edu.cn (F.W.); xbyang@scnu.edu.cn (X.Y.) \\ 2 School of Civil Engineering, Zhengzhou University, Zhengzhou 450001, China; brightgalahad@gmail.com \\ * Correspondence: wzc@scnu.edu.cn; Tel.: +86-1311-955-1688
}

\section{check for} updates

Citation: Qin, S.; Huang, H.; Jie, K.; Zeng, S.; Chen, L.; Liu, H.; Guo, J.; Meng, H.; Wang, F.; Yang, X.; et al. Active Modulating the Intensity of Bifocal Metalens with Electrically Tunable Barium Titanate (BTO) Nanofins. Nanomaterials 2021, 11 , 2023. https://doi.org/10.3390/ nano11082023

Academic Editor: Antonio Di Bartolomeo

Received: 9 July 2021

Accepted: 4 August 2021

Published: 8 August 2021

Publisher's Note: MDPI stays neutral with regard to jurisdictional claims in published maps and institutional affiliations.

Copyright: (c) 2021 by the authors. Licensee MDPI, Basel, Switzerland. This article is an open access article distributed under the terms and conditions of the Creative Commons Attribution (CC BY) license (https:/ / creativecommons.org/licenses/by/ $4.0 /)$.

\begin{abstract}
The multifocal metalens with an adjustable intensity has great potential in many applications such as the multi-imaging system, but it is less studied. In this paper, by combining the electro-optic material barium titanate (BTO) with the Pancharatnam-Berry phase, an electrically modulated bifocal metalens in a visible light band is innovatively proposed. Due to the electro-optic effect, we can control the refractive index of the BTO nanofins to vary between 2.4 and 3.07 by applying different voltages $(0-60 \mathrm{~V})$. Thus, the method of modulating the intensity ratio of the two focal points is applying an electric field. It is different from using phase change materials or changing the ellipticity of incident light, the strategies proposed in previous studies. Moreover, when the applied voltage is $0 \mathrm{~V}$ or $60 \mathrm{~V}$, the bifocal metalens becomes a single focal metalens with different focal lengths, and the full width at half maximum of each focal point is close to the diffraction limit. It has great potential in applications of optical storage, communication and imaging systems.
\end{abstract}

Keywords: bifocal metalens; barium titanate; electro-optic

\section{Introduction}

In the past decades, metalens has drawn intensive attention due to the ability to control the wavefront to focus with a small footprint based on methods of realizing the discontinue phase [1-10]. However, tremendous researches mainly concentrated on focusing with one focal point; the bifocal metalens or multifocal metalens with great potential in multiimaging and micro-manipulating optics is less studied. With arrays of inhomogeneous optical scatters placed on one or more thin surfaces, the bifocal metalens allows one beam of incident light to focus at two different focal points [11-15]. As a member of metalens, the bifocal metalens also exists with the problem of being static in nature. Therefore, the modulation of bifocal metalens is also a significant topic, especially the tunable intensity in focal points.

Up to now, methods to realize the adjustable intensity of the bifocal metalens have been limited. One important method is combining thermally controlled phase change materials with the bifocal metalens [16-18]. Among them, $\mathrm{Ge}_{2} \mathrm{Sb}_{2} \mathrm{Te}_{5}$ (GST) is the most representative material for the application of the adjustable bifocal metalens. GST is a kind of phase change material that has a significant refractive index shift and absorption difference in different states [19-21]. Most of the methods based on GST rely on dividing the metalens into different areas. The design will lead to a low signal-to-noise ratio [17,22]. Moreover, the fractional crystallization of GST is difficult to control [23] which impedes the development of a continuous precise regulation. Additionally, GST is not applicable to a metalens in the visible band with a large absorption in both states. Another phase change 
material used in the bifocal metalens is $\mathrm{VO}_{2}$ which works in the infrared band [24] and terahertz [18]. Similar to GST, it is also not suitable for the realization of the metalens in the visible band.

Except for phase change materials, a major method to modulate the bifocal metalens is altering the polarization of the incident beam [25-28]. For instance, Shengnan Tian et al., proposed a dielectric longitudinal bifocal metalens with an adjustable intensity [26]. By entering different elliptically polarized light, the method can realize adjusting the relative intensity of the two focal points flexibly. However, the change of incident light may bring inconvenience in some conditions and the response time is not fast enough. An adjustable intensity, high-speed modulation and constant light source are the ideal scenarios for the bifocal metalens in practical application.

In this paper, we propose an electrically modulated bifocal metalens whose intensity can be adjusted based on the electro-optical material barium titanate (BTO) in the visible region with high-speed modulation. Due to the Pockels effect, the refractive index of BTO can be modulated by varying the applied voltage [29]. Moreover, BTO fits the visible region that can make up for the problem of the working region of phase change materials. As shown in Figure 1, by designing a metalens doublet to combine the multilayer Pancharatnam-Berry phase with BTO, the relative intensity ratio of the two foci can be adjusted. The double-layer structure can realize the unified control of the metalens. When adjusting the intensity, the metalens just needs one applied voltage. It effectively avoids the complexity of applying different controls simultaneously on a micro-nano structure. The upper layer of the metalens is composed of BTO nanofins. The bottom layer is composed of Si nanofins. Additionally, because of the thin thickness between the two layers, to simplify the production in reality, the $\mathrm{Si}$ nanofins layer is filled with $\mathrm{SiO}_{2}$. In order to realize the arbitrary intensity ratio, the intensity of each focus should be able to change between 0 and the maximum value. The numerical simulations are completed based on the commercial software finite-difference time-domain (FDTD) Solutions 2018a (Lumerical Inc., Vancouver, BC, Canada). The results show that the metalens can focus on a single focal point when $n=2.4$ $\left(\mathrm{F}_{1}=9 \mu \mathrm{m}\right)$ and $n=3.07\left(\mathrm{~F}_{2}=15 \mu \mathrm{m}\right)$ to realize the varying of the focal length. The simulated focal lengths correspond to the theoretical values well. Additionally, the full width at half maximums (FWHMs) are close to the diffraction limited values. Then, we gradually increase the refractive index of BTO from 2.4 to 3.07. Values of the intensity ratio between the two foci show that the design has the ability to modulate the metalens flexibly. Additionally, simulated focal lengths and FWHMs show that the image quality under the bifocal condition is still high. We believe the proposed method will provide a potential platform for multi-imaging systems, optical free-space communication and optical data storage.

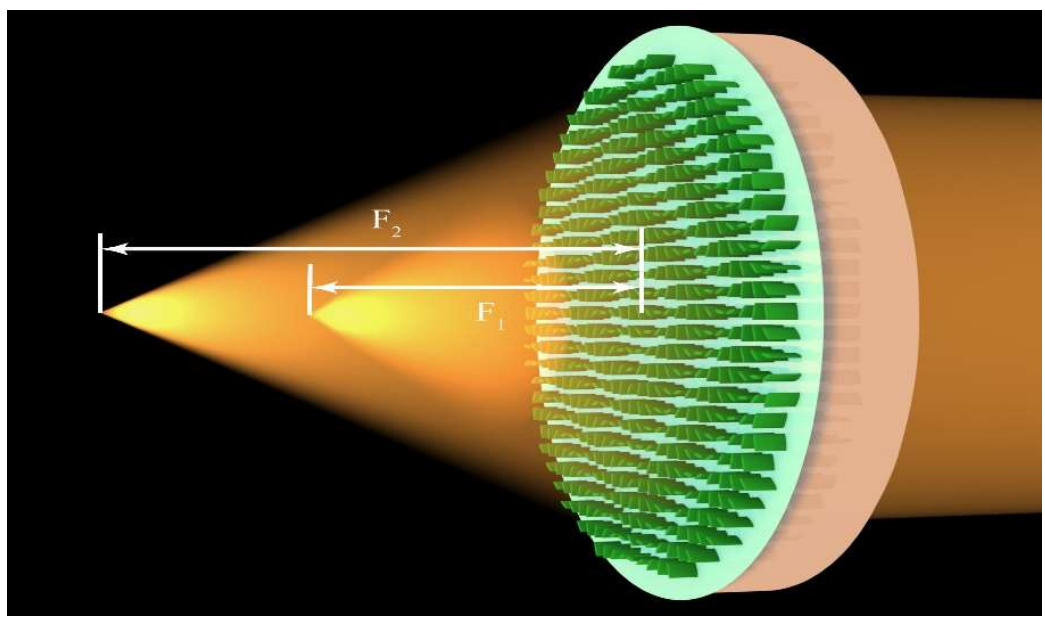

Figure 1. Schematic of the electrically modulated bifocal metalens. The metalens can focus at two different focal points at the same time. By applying different voltages, the intensity ratio of the two focal points changes. 


\section{Materials and Methods}

The metalens is composed of BTO nanofins layer, Si nanofins layer, indium tin oxide (ITO) layer and $\mathrm{SiO}_{2}$ substrate. As a kind of electro-optic crystal, BTO has an excellent ability to alter the refractive index. The shift of the refractive index is proportional to applied electric field because of the electro-optic effect named Pockels effect. Meanwhile, BTO is chemically and thermally stable and has ultrafast modulation speed (sub-ps) [29,30]. Additionally, compared with other electric-optical materials such as $\mathrm{LiNbO}_{3}$ [31], the Pockels coefficient of BTO is much larger. Therefore, for the same refractive index shift, the applied electric field on BTO is lower. Moreover, its working bandwidth can cover visible and near-infrared region that solves the absorption problem of phase change materials in visible region. The ordinary refractive index of BTO will shift with the application of the voltage as follows [32,33]:

$$
\mathrm{n}=\mathrm{n}_{0}+\frac{1}{2} \mathrm{n}_{0}^{3} \mathrm{r}_{51} \mathrm{~V} / \mathrm{t}_{\mathrm{D}}
$$

where $\mathrm{n}_{0}$ is the real part of the refractive index of BTO with no application of electric field, the electro-optic (EO) coefficient $r_{51}$ is $1300 \mathrm{pm} / \mathrm{V}$ according to the past research. $V$ is the applied voltage to produce refractive index shift and $t_{D}$ is thickness of the BTO layer which means the height of BTO nanofins. In the proposed design, the height of BTO nanofins was set as $800 \mathrm{~nm}$. Therefore, by applying a voltage that was lower than $60 \mathrm{~V}$, the refractive index can shift from 2.4 to 3.07. The function of the ITO layer was to apply voltage from the bottom of BTO layer which makes the metalens easy to be compacted. Moreover, as shown in Figure 1, if the thickness between the Si layer and BTO layer has a relatively large value, it will influence the phase distribution. Therefore, the thickness was set as $0.4 \mu \mathrm{m}$, a little larger than half of the wavelength to obtain a better result. In order to avoid the manufacturing problem due to the thickness being so thin, $\mathrm{SiO}_{2}$ filled in the $\mathrm{Si}$ layer as filling material [34].

As shown in Figure 2, the unit cell is a double-layer structure. According to multilayer Pancharatnam-Berry $(\mathrm{PB})$ phase method, when a circularly polarized beam incidents to the cell, the Jones matrix can be expressed as [34]:

$$
\begin{gathered}
\mathrm{J}\left(\theta_{1}, \theta_{2}\right)=\left[\begin{array}{cc}
\frac{1}{4} \mathrm{~T}_{1} \mathrm{~T}_{1}^{\prime}+\frac{1}{4} \mathrm{~T}_{2} \mathrm{~T}_{2}^{\prime} \mathrm{e}^{\mathrm{j} 2\left(\theta_{1}-\theta_{2}\right)} & \frac{1}{4} \mathrm{~T}_{2} \mathrm{~T}_{1}^{\prime} \mathrm{e}^{\mathrm{j} 2 \theta_{1}}+\frac{1}{4} \mathrm{~T}_{1} \mathrm{~T}_{2}^{\prime} \mathrm{e}^{\mathrm{j} 2 \theta_{2}} \\
\frac{1}{4} \mathrm{~T}_{2} \mathrm{~T}_{1}^{\prime} \mathrm{e}^{-\mathrm{j} 2 \theta_{1}}+\frac{1}{4} \mathrm{~T}_{1} \mathrm{~T}_{2}^{\prime} \mathrm{e}^{-\mathrm{j} 2 \theta_{2}} & \frac{1}{4} \mathrm{~T}_{1} \mathrm{~T}_{1}^{\prime}+\frac{1}{4} \mathrm{~T}_{2} \mathrm{~T}_{2}^{\prime} \mathrm{e}^{\mathrm{j} 2\left(\theta_{2}-\theta_{1}\right)}
\end{array}\right] \\
\begin{cases}\mathrm{t}_{\mathrm{o}}+\mathrm{t}_{\mathrm{e}}=\mathrm{T}_{1} & \mathrm{t}_{\mathrm{o}}-\mathrm{t}_{\mathrm{e}}=\mathrm{T}_{2} \\
\mathrm{t}_{\mathrm{o}}{ }^{\prime}+\mathrm{t}_{\mathrm{e}}{ }^{\prime}=\mathrm{T}_{1}{ }^{\prime} & \mathrm{t}_{\mathrm{o}}{ }^{\prime}-\mathrm{t}_{\mathrm{e}}{ }^{\prime}=\mathrm{T}_{2}^{\prime}\end{cases}
\end{gathered}
$$

in each unit cell doublet, $\theta_{1}$ and $\theta_{2}$ are rotation angles of BTO nanofin and Si nanofin. $t_{0}, t_{e}$ (BTO nanofin) and $\mathrm{t}_{\mathrm{o}}{ }^{\prime}, \mathrm{t}_{\mathrm{e}}{ }^{\prime}$ (Si nanofin) represent the complex transmission coefficients along ordinary $\left(l_{1}, l_{2}\right)$ and extraordinary $\left(\mathrm{w}_{1}, \mathrm{w}_{2}\right)$ axes. For the proposed metalens, the incident beam was right-handed circularly polarized. Therefore, the output beam follows the result:

$$
\mathrm{E}_{\text {out }}=\frac{1}{4} \mathrm{~T}_{1} \mathrm{~T}_{1}^{\prime}\left(\begin{array}{c}
1 \\
\mathrm{i}
\end{array}\right)+\frac{1}{4} \mathrm{~T}_{2} \mathrm{~T}_{2}^{\prime} \mathrm{e}^{\mathrm{j} 2\left(\theta_{1}-\theta_{2}\right)}\left(\begin{array}{c}
1 \\
\mathrm{i}
\end{array}\right)+\frac{1}{4} \mathrm{~T}_{2} \mathrm{~T}_{1}^{\prime} \mathrm{e}^{-\mathrm{j} 2 \theta_{1}}\left(\begin{array}{c}
1 \\
-\mathrm{i}
\end{array}\right)+\frac{1}{4} \mathrm{~T}_{1} \mathrm{~T}_{2}^{\prime} \mathrm{e}^{-\mathrm{j} 2 \theta_{2}}\left(\begin{array}{c}
1 \\
-\mathrm{i}
\end{array}\right)
$$

In order to realize the condition that the relative intensity ratio between the two foci can cover all values, the metalens should have the ability to focus on one point for extreme condition. It means the ratio can be close to $1 / 0$ or $0 / 1$. First, the Si nanofin works as a half-wave plate which means $\mathrm{T}_{1}^{\prime}=0$ to decrease the resulting phase distributions:

$$
\mathrm{E}_{\text {out }}=\frac{1}{4} \mathrm{~T}_{2} \mathrm{~T}_{2}^{\prime} \mathrm{e}^{\mathrm{j} 2\left(\theta_{1}-\theta_{2}\right)}\left(\begin{array}{c}
1 \\
\mathrm{i}
\end{array}\right)+\frac{1}{4} \mathrm{~T}_{1} \mathrm{~T}_{2}^{\prime} \mathrm{e}^{-\mathrm{j} 2 \theta_{2}}\left(\begin{array}{c}
1 \\
-\mathrm{i}
\end{array}\right)
$$


(a)

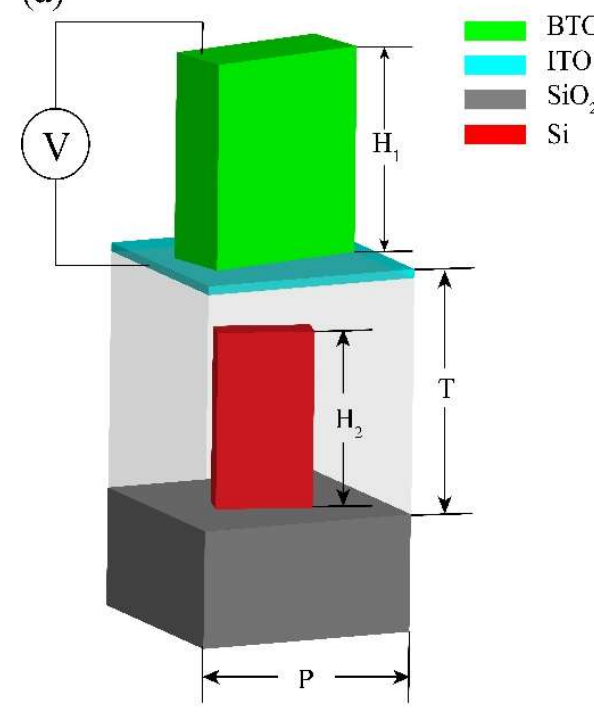

(b)

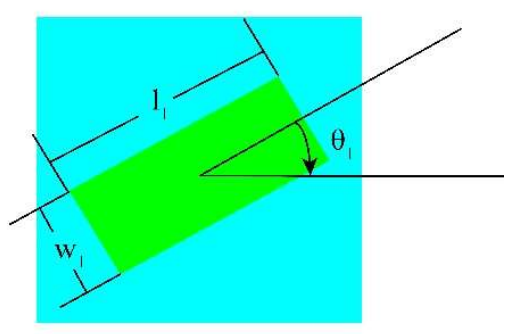

(c)

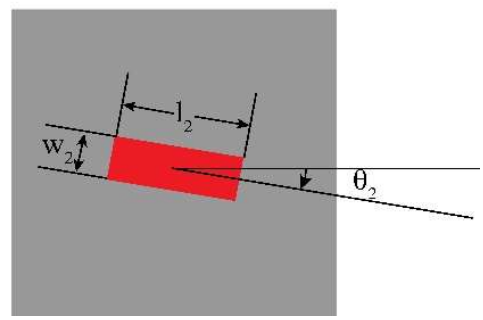

Figure 2. (a) Diagrams of the unit cell, the upper layer is BTO nanofin and the bottom layer is $\mathrm{Si}$ nanofin. $(\mathbf{b}, \mathbf{c})$ represent the schematic diagrams of the upper layer and lower layer rotation angles. The parameters of BTO nanofin and Si nanofin are $\mathrm{l}_{1}, \mathrm{w}_{1}, \mathrm{H}_{1}$ and $\mathrm{l}_{2}, \mathrm{w}_{2}, \mathrm{H}_{2}$, respectively. $\theta_{1}$ and $\theta_{2}$ are rotation angles of BTO nanofin and Si nanofin. $\mathrm{P}$ is the period of the unit cell. $\mathrm{T}$ is the distance between the two layers.

Based on the simplified equation, the function of the BTO nanofin has to be able to switch between a half-wave plate $\left(T_{1}=0\right)$ and a full-wave plate $\left(T_{2}=0\right)$ when the refractive index switches between 2.4 and 3. Therefore, when $n=2.4$ and 3.07, the output light follows the equation:

$$
\left\{\begin{array}{l}
\mathrm{E}_{\mathrm{n}=2.4}=\frac{1}{4} \mathrm{~T}_{2} \mathrm{~T}_{2}^{\prime} \mathrm{e}^{\mathrm{j} 2\left(\theta_{1}-\theta_{2}\right)}\left(\begin{array}{c}
1 \\
\mathrm{i}
\end{array}\right) \\
\mathrm{E}_{\mathrm{n}=3.07}=\frac{1}{4} \mathrm{~T}_{1} \mathrm{~T}_{2}^{\prime} \mathrm{e}^{-\mathrm{j} 2 \theta_{2}}\left(\begin{array}{c}
1 \\
-\mathrm{i}
\end{array}\right)
\end{array}\right.
$$

Above all, in this paper, when there was no application of voltage, the BTO nanofin worked as a half-wave plate with the imparted phase distribution of the output light equaling $2\left(\theta_{1}-\theta_{2}\right)$. After applying voltage until the refractive index reached 3.07, the inserted phase distribution became $-2 \theta_{2}$. When the refractive index was between 2.4 and 3.07 , the phase distribution of the outgoing light was divided into two parts. One part was $E_{n}=2.4$, the other part was $E_{n}=3.07$. Additionally, as the refractive index changes, the ratio of the two inserted phase distributions will also change.

\section{Optimization of Nanofins}

The desired parameters of the BTO and Si nanofins were calculated by a threedimensional FDTD method (Lumerical Inc., Vancouver, BC, Canada). The mesh grids were $10 \mathrm{~nm} \times 10 \mathrm{~nm} \times 10 \mathrm{~nm}$. For the $x$ - and $y$-axis, the periodic boundary condition was applied and the boundary condition for the z-axis was a perfectly matched layer (PML). The working wavelength was set to $600 \mathrm{~nm}$ to verify the proposed method. By changing the lengths of the ordinary axes $\left(l_{1}, l_{2}\right)$ and the extraordinary axes $\left(\mathrm{w}_{1}, \mathrm{w}_{2}\right)$ of the BTO nanofins $\left(\mathrm{n}_{0}=2.4\right)$ and the Si nanofins with a period of $350 \mathrm{~nm} \times 350 \mathrm{~nm}$, the phase shift difference between the linearly polarized beam along the ordinary axes (x-polarized) and the extraordinary axes (y-polarized) can be seen in Figure 3. From Figure 3, points A and B with a phase shift difference $\pi$, which means the BTO nanofin $\left(n_{0}=2.4\right)$ and the $\mathrm{Si}$ nanofin can work as a half-wave plate, were the desired parameters. Therefore, parameters of the BTO nanofins and the Si nanofins were confirmed as $l_{1}=260 \mathrm{~nm}, \mathrm{w}_{1}=110 \mathrm{~nm}$, 
$\mathrm{H}_{1}=800 \mathrm{~nm}$ and $\mathrm{l}_{2}=140 \mathrm{~nm}, \mathrm{w}_{2}=50 \mathrm{~m}, \mathrm{H}_{2}=600 \mu \mathrm{m}$, respectively. Then, the refractive index of $\mathrm{BTO}$ was changed to find a value that would meet the condition $\mathrm{T}_{2}=0$.

(a)

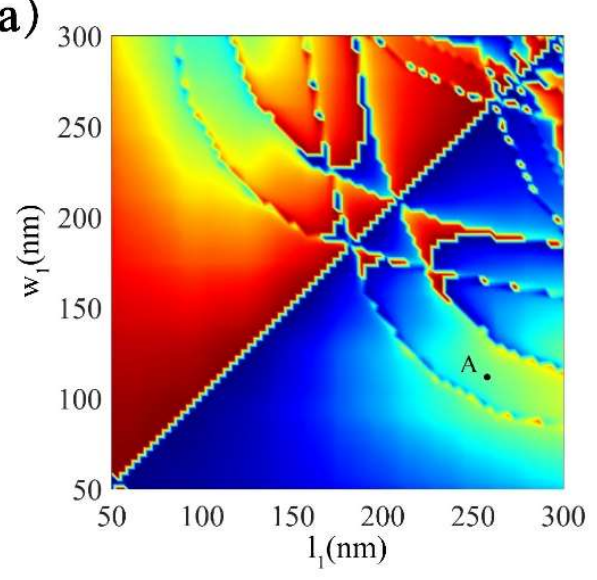

(b)

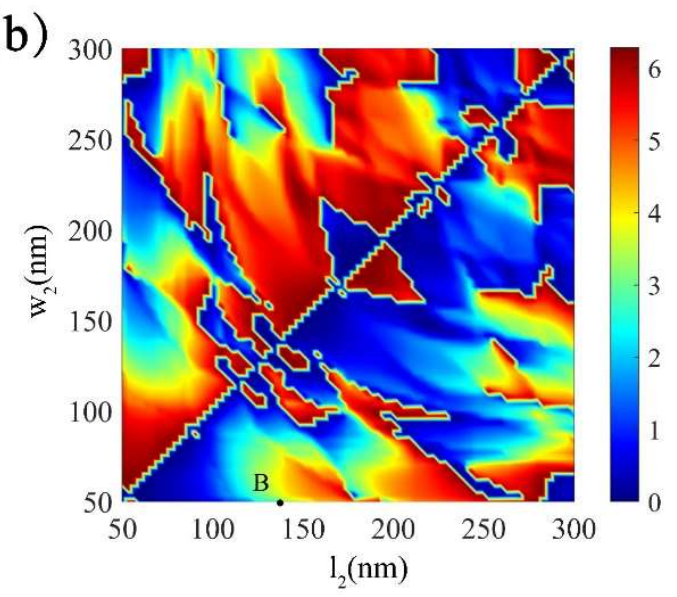

Figure 3. The phase shift difference $(\Delta \varphi)$ of linearly polarized light along ordinary axes and extraordinary axes with different lengths of ordinary axes $\left(l_{1}, l_{2}\right)$ and extraordinary axes $\left(\mathrm{w}_{1}, \mathrm{w}_{2}\right)$ for $(\mathbf{a})$ BTO nanofin and (b) Si nanofin.

As shown in Figure 4a, for the BTO nanofins, the phase shift of the linearly polarized beam along the ordinary axes (x-polarized) and the extraordinary axes (y-polarized) and the phase shift difference between the output light of the two beams changed with the refractive index as depicted. The results showed that when the refractive index of the BTO layer was 2.4 (no applied voltage), the phase shift difference was $\pi$. During the period of increasing the refractive index to 3.07, the phase shift difference could cover all values from $0-\pi$, and become 0 when $n=3.07$. In order to further show that the optimization results met the design requirements, the polarization conversion efficiency (PCR) of the BTO nanofins ( $n=2.4$ and 3.07) and the Si nanofins from $500 \mathrm{~nm}$ to $700 \mathrm{~nm}$ are depicted in Figure $4 \mathrm{~b}$. The results showed that the PCR of the BTO nanofins can reach $98.4 \%(n=2.4)$ and $0.3 \%(n=3.07)$ when the wavelength was $600 \mathrm{~nm}$. Therefore, the resultant parameters can fit the desired functions well.

(a)

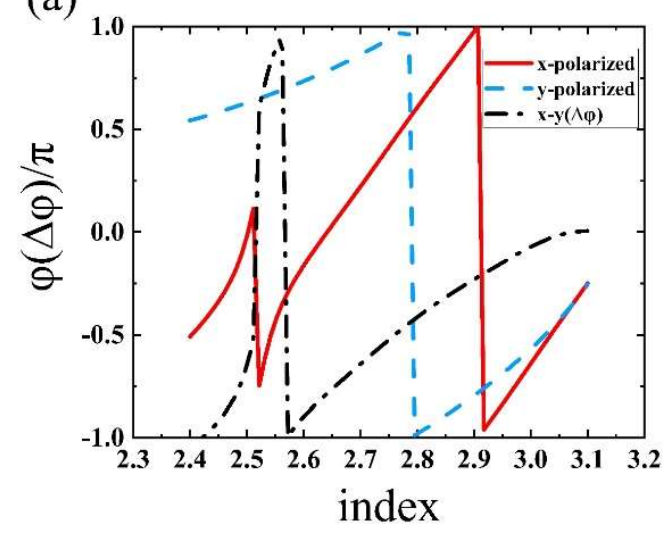

(b)

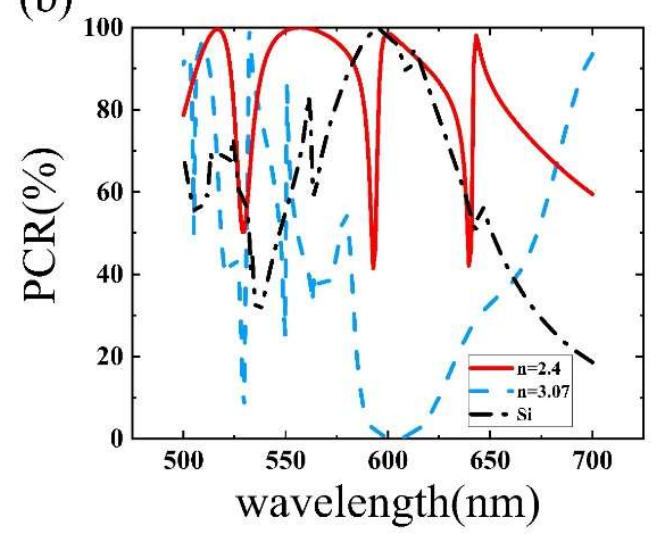

Figure 4. (a) Phase shift of the linearly polarized beam along ordinary axes (x-polarized) and extraordinary axes (y-polarized) and phase shift difference between them when the refractive index of the BTO nanofin changes from 2.4 to 3.1. (b) PCR of the BTO nanofin $(n=2.4$ and $n=3.07)$ and the Si nanofin. 


\section{Results and Discussion}

Based on all above analyses, a two-dimensional bifocal metalens was proposed. The phase profile to focus the incident beam had to meet the equation [34]:

$$
\varphi_{\mathrm{f}}=\frac{2 \pi}{\lambda}\left(\mathrm{f}-\sqrt{\mathrm{x}^{2}+\mathrm{y}^{2}+\mathrm{f}^{2}}\right)
$$

where $\lambda$ is the working wavelength, $f$ is the focal length and $x$ and $y$ are the discretized spatial coordinates. At last, in order to realize the proposed design, the rotation angle of the BTO nanofins and the Si nanofins should meet the equation as follows:

$$
\left\{\begin{array}{c}
2\left(\theta_{1}-\theta_{2}\right)=\varphi_{\mathrm{f}_{1}} \\
-2 \theta_{2}=\varphi_{\mathrm{f}_{2}}
\end{array}\right.
$$

In the proposed bifocal metalens, the incident beam was right-handed circularly polarized. The wavelength was $600 \mathrm{~nm}$. The radius of the metalens was set as $10 \mu \mathrm{m}$. To show the proposed design, we set the two focal lengths as $f_{1}=9 \mu \mathrm{m}$ and $f_{2}=15 \mu \mathrm{m}$. The discussion of results is listed in Sections 4.1 and 4.2

\subsection{Switch of Single Focal Point}

Firstly, in order to verify that the relative intensity of the two focal points can cover the extreme condition, we showed that the focal length of the metalens can switch between $\mathrm{f}_{1}$ and $\mathrm{f}_{2}$ in Figure 5. When there was no application of voltage, the refractive index of BTO was 2.4. The theoretical focal length was $\mathrm{f}_{1}$. After the voltage reached $60 \mathrm{~V}$, the refractive index of BTO was 3.07. The theoretical focal length became $f_{2}$. According to Figure 5a, $d$, the intensity profiles of the two conditions on the $x-z$ plane were shown. The simulated focal lengths of BTO with $n=2.4$ and $n=3.07$ were $9.43 \mu \mathrm{m}$ and $15.13 \mu \mathrm{m}$ which were close to the design values. Figure $5 b$,e shows the intensity distribution of the first focal point and the second focal point on the $x-y$ plane. Furthermore, in Figure $5 c, f$, to show the calculated spot size of the two focal points, we calculated the full width at half maximum (FWHM) to compare with the diffraction limited value according to the formula $\lambda / 2 \mathrm{NA}$, where the equation of the numerical aperture NA was $N A=\sin \left[\tan ^{-1}(\mathrm{D} / 2 \mathrm{f})\right]$. When the diameter D of the metalens was $20 \mu \mathrm{m}$, the diffraction limited values of the two focal points were about $404 \mathrm{~nm}$ and $541 \mathrm{~nm}$. The simulated FWHMs were $422 \mathrm{~nm}$ and $568 \mathrm{~nm}$ which corresponded to the theoretical diffraction limited values. For each focal point, on the focal plane, a circular area with a radius that is three times of its FWHM was picked. Then, the ratio of the optical power in this area to the power of the incident light was calculated to obtain the focusing efficiency [35]. The focusing efficiencies of the two focal points were about $46.60 \%$ and $40.15 \%$ when the refractive index was 2.4 and 3.07. The efficiency difference of the two foci may have mainly come from different numerical apertures (NAs). 

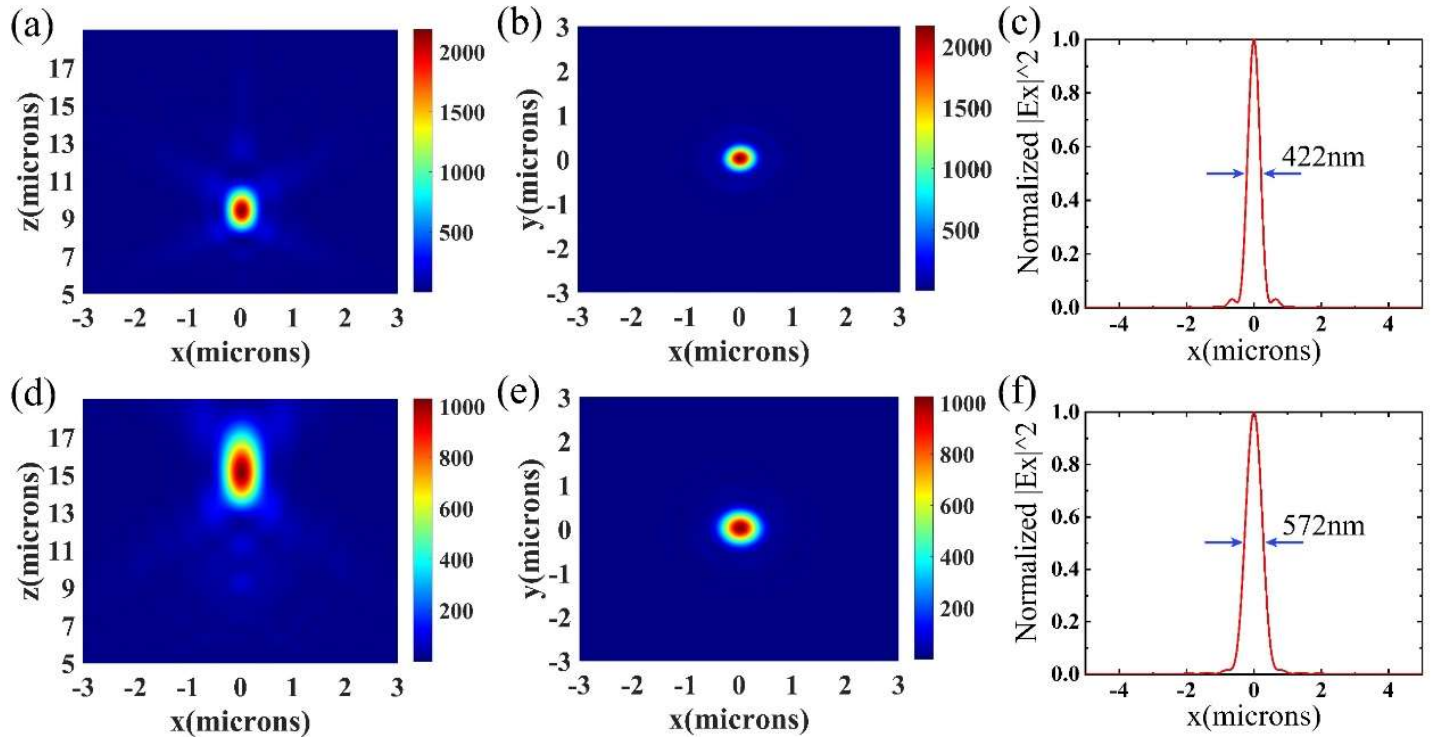

Figure 5. The simulated results for the refractive index of BTO was 2.4 and 3.07. (a,d) The intensity distribution of the first focal point (when $n=2.4$ ) and the second focal point (when $n=3.07)$ on the $x-z$ plane; $(\mathbf{b}, \mathbf{e})$ the intensity distribution of the first focal point and the second focal point on the $x-y$ plane; $(\mathbf{c}, \mathbf{f})$ the full width at half-maxima of the first focal point $\left(f_{1}\right)$ and second focal point $\left(\mathrm{f}_{2}\right)$ were $422 \mathrm{~nm}$ and $572 \mathrm{~nm}$.

\subsection{Bifocal Metalens with Tunable Intensity Ratio}

In order to demonstrate that the intensity ratio between the two focal points of the designed metalens can be modulated, the refractive index of BTO was set as 2.6, 2.7, 2.8 and 2.9 (with applied voltages of $18 \mathrm{~V}, 27 \mathrm{~V}, 36 \mathrm{~V}$ and $45 \mathrm{~V}$ ). In Figure 6, the intensity distributions of the simulated results are shown. Combined with Figure $5 \mathrm{a}, \mathrm{d}$, the results showed that when the refractive index of BTO gradually increased, the intensity of the first focal point (with focal length $\mathrm{f}_{1}=9 \mu \mathrm{m}$ ) would gradually decrease until the focal point disappeared. Additionally, the intensity of the second focal point (with focal length $\mathrm{f}_{2}=15 \mu \mathrm{m}$ ) showed a trend of a gradual increase.

Figure 7 shows the simulated results of the intensity along the $z$-axis $(x=0, y=0)$. Additionally, Table 1 makes a summary of the focusing performance of the bifocal metalens with a different refractive index. The results show that the FWHM of each focal point was close to its theoretical diffraction limit. Additionally, the simulated focal lengths all met the designed values. Because for the one focal point case the position of the other focal point had no focal point, the FWHM and focal length were not calculated. Moreover, according to the intensity ratio, the ratio could reach $1 / 0.01$ and $0.08 / 1$ in the extreme condition with $n=2.4$ and 3.07 (we chose the intensity at the theoretical focal point as the intensity of the disappearing focal point). The difference also came from the different NAs. The values were near the desirable values $1 / 0$ and $0 / 1$. When the refractive index increased from 2.4 to 3.07 , the variation trend of the intensity ratio presented a process of gradual change, which means the design can alter the ratio flexibly. 
(a)

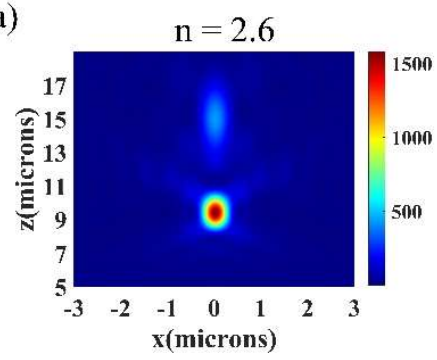

(c)

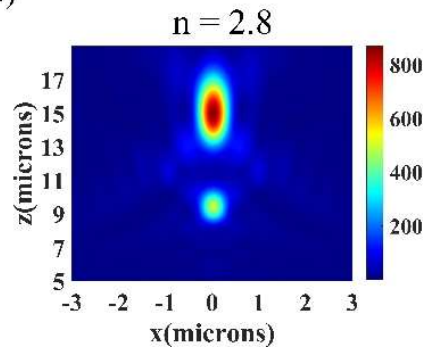

(b)

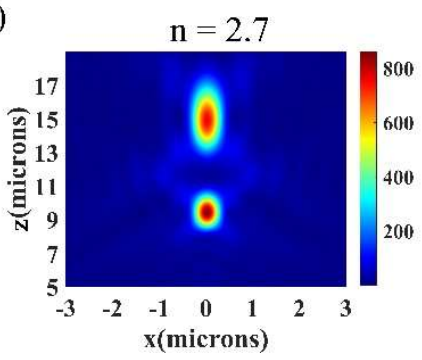

(d)

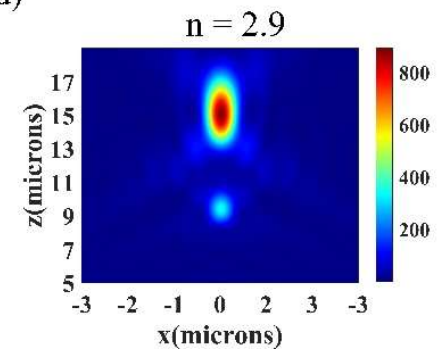

Figure 6. The intensity distribution of the bifocal metalens on the $x-z$ plane when the refractive index of BTO was 2.6, 2.7, 2.8 and 2.9.

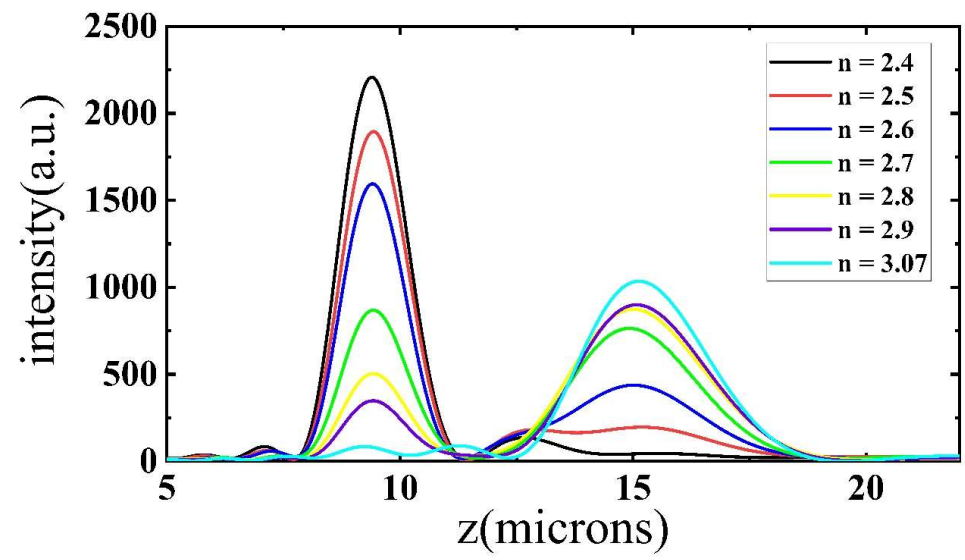

Figure 7. Simulated intensity results along z-axis when the refractive index of BTO changed.

Table 1. The focusing performance of the bifocal metalens with different refractive index.

\begin{tabular}{|c|c|c|c|c|c|c|c|}
\hline & $n=2.4$ & $n=2.5$ & $n=2.6$ & $n=2.7$ & $n=2.8$ & $n=2.9$ & $n=3.07$ \\
\hline \multirow{2}{*}{ Simulated value $(\mu \mathrm{m})$} & 9.43 & 9.43 & 9.40 & 9.43 & 9.43 & 9.43 & - \\
\hline & 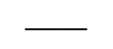 & 15.20 & 15.02 & 14.93 & 15.00 & 15.09 & 15.13 \\
\hline \multirow{2}{*}{ FWHM (nm) } & 422 & 429 & 432 & 431 & 437 & 445 & - \\
\hline & 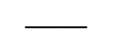 & 563 & 564 & 568 & 563 & 567 & 568 \\
\hline Intensity ratio $\left(\mathrm{f}_{1}: \mathrm{f}_{2}\right)$ & $1 / 0.01$ & $1 / 0.1$ & $1 / 0.3$ & $1 / 0.9$ & $0.6 / 1$ & $1 / 0.4$ & $0.08 / 1$ \\
\hline
\end{tabular}

\section{Conclusions}

In summary, we proposed a high-speed electrically modulated bifocal metalens with an adjustable intensity based on the EO material BTO in the visible region. The intensity ratio of the two focal points could be adjusted by applying different voltages of 0 to $60 \mathrm{~V}$. When there was no application of voltage, the metalens could only focus on the first focal point (focal length is $\mathrm{f}_{1}$ ). As the applied voltage increased, the intensity of the first focal point gradually decreased. Meanwhile, the intensity of the second focal point (focal length is $f_{2}$ ) increased with the voltage. Until the voltage reached $60 \mathrm{~V}$, the design 
worked as a single-focusing metalens again with a focal length $\mathrm{f}_{2}$. Different from previous methods, the metalens realized through the intensity ratio between two different focal points can be adjusted arbitrarily with a constant optical source in the visible light band. In addition, when the voltage switched between $0 \mathrm{~V}$ and $60 \mathrm{~V}$, the metalens could work as a varifocal metalens with two different focal lengths. The proposed electrically modulated metalens has great potential in the application of detectors, optical storage, laser printing and multi-functional devices.

Author Contributions: Conceptualization, S.Q.; methodology, S.Q.; software, S.Q. and S.Z. validation, S.Q. and Z.W.; formal analysis, Z.W., J.G., F.W., H.M., H.L., K.J., H.H. and L.C.; investigation, S.Q. and Z.W.; data curation, H.H. and S.Z.; writing-original draft preparation, K.J.; writing-review and editing, Z.W., J.G., F.W., H.M., H.L., K.J., X.Y. and L.C.; visualization, S.Z.; supervision, Z.W.; project administration, Z.W.; funding acquisition, Z.W. and H.L. All authors have read and agreed to the published version of the manuscript.

Funding: This research was funded by the National Natural Science Foundation of China (NSFC) under grant nos. 61774062, 61875057 and the Natural Science Foundation of Guangdong Province under grant nos. 2021A1515010352.

Data Availability Statement: No new data were created or analyzed in this study. Data sharing is not applicable to this article.

Acknowledgments: The Science and Technology Program of Guangzhou (No. 2019050001).

Conflicts of Interest: The authors declare no conflict of interest.

\section{References}

1. Yin, L.; Vlasko-Vlasov, V.K.; Pearson, J.; Hiller, J.M.; Hua, J.; Welp, U.; Brown, D.E.; Kimball, C.W. Subwavelength focusing and guiding of surface plasmons. Nano Lett. 2005, 5, 1399-1402. [CrossRef]

2. Khorasaninejad, M.; Chen, W.T.; Devlin, R.C.; Oh, J.; Zhu, A.Y.; Capasso, F. Metalenses at visible wavelengths: Diffraction-limited focusing and subwavelength resolution imaging. Science 2016, 352, 1190-1194. [CrossRef]

3. Wang, S.; Wu, P.C.; Su, V.C.; Lai, Y.C.; Chen, M.K.; Kuo, H.Y.; Chen, B.H.; Chen, Y.H.; Huang, T.T.; Wang, J.H. A broadband achromatic metalens in the visible. Nat. Nanotechnol. 2018, 13, 227-232. [CrossRef]

4. Chen, C.; Song, W.; Chen, J.W.; Wang, J.H.; Chen, Y.H.; Xu, B.; Chen, M.K.; Li, H.; Fang, B.; Chen, J. Spectral tomographic imaging with aplanatic metalens. Light Sci. Appl. 2019, 8, 99. [CrossRef] [PubMed]

5. Presutti, F.; Monticone, F. Focusing on bandwidth: Achromatic metalens limits. Optica 2020, 7, 624-631. [CrossRef]

6. Balli, F.; Sultan, M.; Lami, S.K.; Hastings, J.T. A hybrid achromatic metalens. Nat. Commun. 2020, 11, 3892. [CrossRef] [PubMed]

7. Li, X.; Wei, S.; Cao, G.; Lin, H.; Zhao, Y.; Jia, B. Graphene metalens for particle nanotracking. Photonics Res. 2020, 8, 1316-1322. [CrossRef]

8. Yoon, G.; Kim, K.; Kim, S.U.; Han, S.; Lee, H.; Rho, J. Printable nanocomposite metalens for high-contrast near-infrared imaging. ACS Nano 2021, 15, 698-706. [CrossRef] [PubMed]

9. Shalaginov, M.Y.; An, S.; Yang, F.; Su, P.; Lyzwa, D.; Agarwal, A.M.; Zhang, H.; Hu, J.; Gu, T. Single-element diffraction-limited fisheye metalens. Nano Lett. 2020, 20, 7429-7437. [CrossRef]

10. Shalaginov, M.Y.; An, S.; Zhang, Y.; Yang, F.; Su, P.; Liberman, V.; Chou, J.B.; Roberts, C.M.; Kang, M.; Rios, C. Reconfigurable all-dielectric metalens with diffraction-limited performance. Nat. Commun. 2021, 12, 1225. [CrossRef]

11. Hashemi, M.; Moazami, A.; Naserpour, M.; Zapata-Rodríguez, C.J. A broadband multifocal metalens in the terahertz frequency range. Opt. Commun. 2016, 370, 306-310. [CrossRef]

12. Chen, Q.; Li, Y.; Han, Y.; Deng, D.; Yang, D.; Zhang, Y.; Liu, Y.; Gao, J. High numerical aperture multifocal metalens based on Pancharatnam-Berry phase optical elements. Appl. Opt. 2018, 57, 7891-7894. [CrossRef] [PubMed]

13. Ma, Y.; Rui, G.; Gu, B.; Cui, Y. Trapping and manipulation of nanoparticles using multifocal optical vortex metalens. Sci. Rep. 2017, 7, 14611. [CrossRef] [PubMed]

14. Zhang, J.; Huang, B.; Yuan, H.; Wang, Z.; Deng, Y.; Wu, J.; Yang, J. Design of multiwavelength confocal plane multifocal metalens based on CGH in visible band. In Proceedings of the 24th National Laser Conference \& Fifteenth National Conference on Laser Technology and Optoelectronics, Shanghai, China, 2 December 2020; p. 1171718.

15. Fan, Q.; Huo, P.; Wang, D.; Liang, Y.; Yan, F.; Xu, T. Visible light focusing flat lenses based on hybrid dielectric-metal metasurface reflector-arrays. Sci. Rep. 2017, 7, 45044. [CrossRef]

16. Li, S.; Zhou, C.; Ban, G.; Wang, H.; Lu, H.; Wang, Y. Active all-dielectric bifocal metalens assisted by germanium antimony telluride. J. Phys. D Appl. Phys. 2019, 52, 095106. [CrossRef]

17. Yin, X.; Steinle, T.; Huang, L.; Taubner, T.; Wuttig, M.; Zentgraf, T.; Giessen, H. Beam switching and bifocal zoom lensing using active plasmonic metasurfaces. Light Sci. Appl. 2017, 6, e17016. [CrossRef] 
18. Kargar, R.; Rouhi, K.; Abdolali, A. Reprogrammable multifocal THz metalens based on metal-insulator transition of VO2-assisted digital metasurface. Opt. Commun. 2020, 462, 125331. [CrossRef]

19. Park, J.W.; Eom, S.H.; Lee, H.; Da Silva, J.L.; Kang, Y.S.; Lee, T.Y.; Khang, Y.H. Optical properties of pseudobinary GeTe, Ge 2 Sb 2 Te 5, GeSb 2 Te 4, GeSb 4 Te 7, and Sb 2 Te 3 from ellipsometry and density functional theory. Phys. Rev. B 2009, 80, 115209. [CrossRef]

20. Bakan, G.; Gerislioglu, B.; Dirisaglik, F.; Jurado, Z.; Sullivan, L.; Dana, A.; Lam, C.; Gokirmak, A.; Silva, H. Extracting the temperature distribution on a phase-change memory cell during crystallization. J. Appl. Phys. 2016, 120, 164504. [CrossRef]

21. Gerislioglu, B.; Bakan, G.; Ahuja, R.; Adam, J.; Mishra, Y.K.; Ahmadivand, A. The role of Ge2Sb2Te5 in enhancing the performance of functional plasmonic devices. Mater. Today Phys. 2020, 12, 100178. [CrossRef]

22. Bai, W.; Yang, P.; Wang, S.; Huang, J.; Chen, D.; Zhang, Z.; Yang, J.; Xu, B. Tunable duplex metalens based on phase-change materials in communication range. Nanomaterials 2019, 9, 993. [CrossRef]

23. Qu, Y.; Li, Q.; Du, K.; Cai, L.; Lu, J.; Qiu, M. Dynamic Thermal Emission Control Based on Ultrathin Plasmonic Metamaterials Including Phase-Changing Material GST. Laser Photonics Rev. 2017, 11, 1700091. [CrossRef]

24. Barker, A., Jr.; Verleur, H.; Guggenheim, H. Infrared optical properties of vanadium dioxide above and below the transition temperature. Phys. Rev. Lett. 1966, 17, 1286. [CrossRef]

25. Chen, X.; Chen, M.; Mehmood, M.Q.; Wen, D.; Yue, F.; Qiu, C.W.; Zhang, S. Longitudinal multifoci metalens for circularly polarized light. Adv. Opt. Mater. 2015, 3, 1201-1206. [CrossRef]

26. Tian, S.; Guo, H.; Hu, J.; Zhuang, S. Dielectric longitudinal bifocal metalens with adjustable intensity and high focusing efficiency. Opt. Express 2019, 27, 680-688. [CrossRef] [PubMed]

27. Mueller, J.B.; Rubin, N.A.; Devlin, R.C.; Groever, B.; Capasso, F. Metasurface polarization optics: Independent phase control of arbitrary orthogonal states of polarization. Phys. Rev. Lett. 2017, 118, 113901. [CrossRef]

28. Chen, Q.; Li, Y.; Liu, Y.; Han, Y. Polarization-multiplexed metalens via spin-independent manipulation of spin-orbit interactions. J. Opt. 2020, 22, 085103. [CrossRef]

29. Abel, S.; Eltes, F.; Ortmann, J.E.; Messner, A.; Castera, P.; Wagner, T.; Urbonas, D.; Rosa, A.; Gutierrez, A.M.; Tulli, D. Large Pockels effect in micro-and nanostructured barium titanate integrated on silicon. Nat. Mater. 2019, 18, 42-47. [CrossRef]

30. Peng, C.; Hamerly, R.; Soltani, M.; Englund, D.R. Design of high-speed phase-only spatial light modulators with two-dimensional tunable microcavity arrays. Opt. Express 2019, 27, 30669-30680. [CrossRef]

31. Bibbò, L.; Khan, K.; Liu, Q.; Lin, M.; Wang, Q.; Ouyang, Z. Tunable narrowband antireflection optical filter with a metasurface. Photonics Res. 2017, 5, 500-506. [CrossRef]

32. Zhu, Q.; Zhang, Y. Defect modes and wavelength tuning of one-dimensional photonic crystal with lithium niobate. Optik 2009, 120, 195-198. [CrossRef]

33. Bibbò, L.; Liu, Q.; Khan, K.; Yadav, A.; Elshahat, S.; Deng, Z.L.; Ouyang, Z. High-speed amplitude modulator with a high modulation index based on a plasmonic resonant tunable metasurface. Appl. Opt. 2019, 58, 2687-2694. [CrossRef] [PubMed]

34. Lin, R.; Li, X. Multifocal metalens based on multilayer Pancharatnam-Berry phase elements architecture. Opt. Lett. 2019, 44, 2819-2822. [CrossRef]

35. Liang, Y.; Liu, H.; Wang, F.; Meng, H.; Guo, J.; Li, J.; Wei, Z. High-efficiency, near-diffraction limited, dielectric metasurface lenses based on crystalline titanium dioxide at visible wavelengths. Nanomaterials 2018, 8, 288. [CrossRef] [PubMed] 